Document downloaded from:

http://hdl.handle.net/10251/65564

This paper must be cited as:

Ferreira Da Silva, AR.; Compañ Moreno, V.; Gonzalez-Meijome, JM. (2015). Reduction in ionic permeability of a silicone hydrogel contact lenses after one month of daily wear. Materials Research Express. 2(6). doi:10.1088/issn.2053-1591.

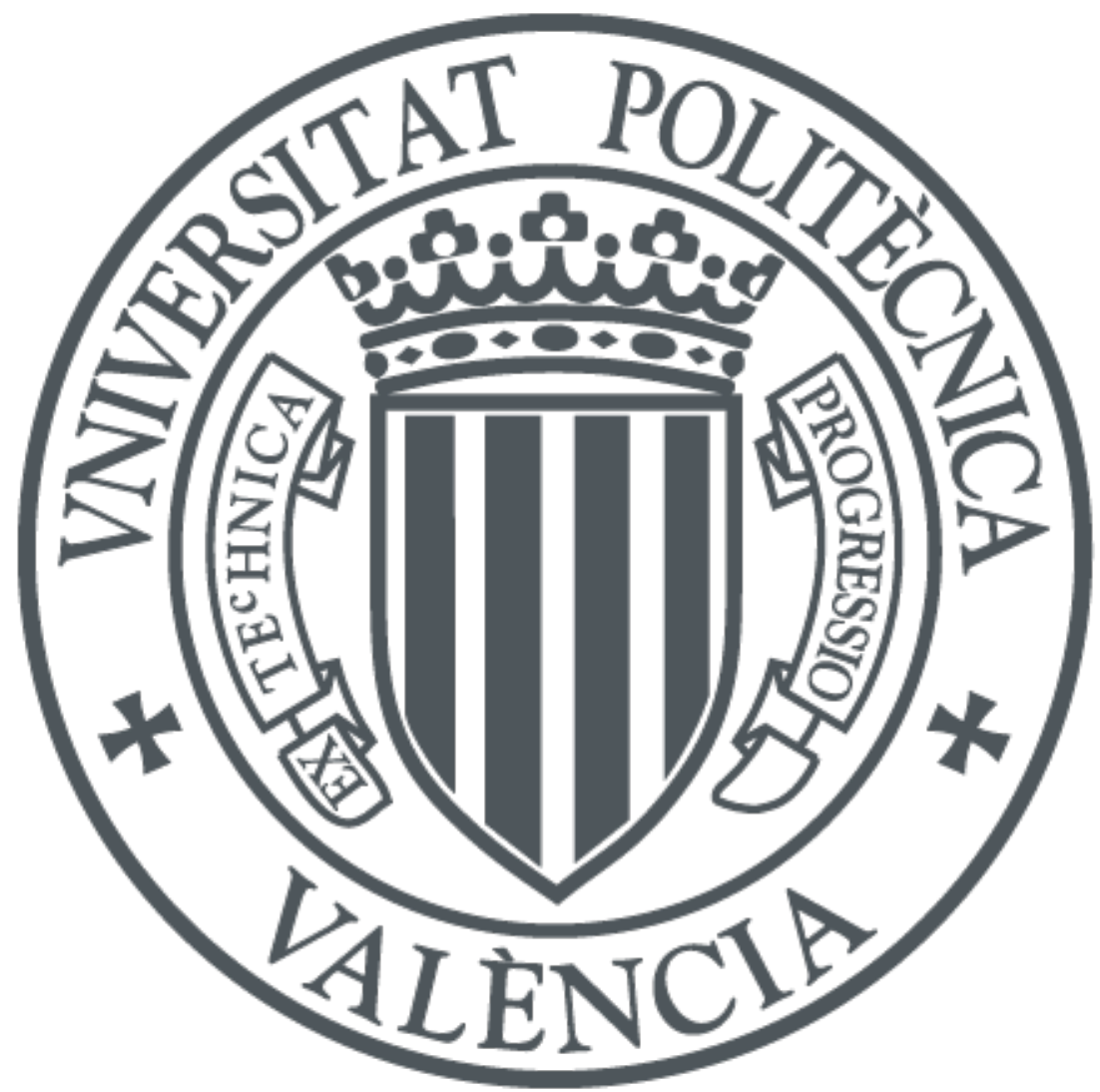

The final publication is available at

http://dx.doi.org/10.1088/2053-1591/2/6/065007

Copyright IOP Publishing

Additional Information 


\section{Reduction in Ionic Permeability of a Silicone Hydrogel Contact Lense after 1 Month of Daily Wear}

Ana Rita Ferreira da Silva, MSc; ${ }^{1}$ Vicente Compañ, PhD; ${ }^{2}$ José M. GonzálezMéijome, $\mathbf{P h D}^{3}$

${ }^{1}$ Clinical and Experimental Optometry Research Laboratory, Center of Physics,

University of Minho, Braga, Portugal

2 Departamento de Termodinámica Aplicada, Universidad Politécnica de Valencia, Spain

Corresponding Author:

José M. González-Méijome, $\mathrm{OD}, \mathrm{PhD}$

Clinical \& Experimental Optometry Research Lab (CEORLab)

Center of Physics (Optometry)

University of Minho

4710-057 Braga - Portugal

Tel.: +351253604072

Fax : +351253604061

e-mail: jgmeijome@fisica.uminho.pt 


\section{ABSTRACT}

Purpose: To compare the ionic permeability using the ionoflux method of new and worn samples of a silicone hydrogel contact lens material.

Methods: An ionoflux experimental setup was established to measure the ionic permeability $(\mathrm{NaCl})$ of soft contact lenses. Samples of a silicone hydrogel lens (Comfilcon A, Coopervision, Pleasanton, CA) with optical powers of $-1.00,-1.50$ and 4.75 diopters (D) were used in this study. Three samples of each power were measured after being worn for 1 month on a daily wear basis. Lenses were cleaned and disinfected every night multipurpose disinfecting solutions. Three samples of new lenses from the same batch were also measured to evaluate the effect of lens wear on the ionic permeability of the lens material. Before measurement, the lenses were previously equilibrated with a solution of $\mathrm{NaCl} 1 \mathrm{M}$ during one week before of each measurement.

Results: The apparent ionic permeability between lenses with -1.00 and $-1.50 \mathrm{D}$ increment $15 \%$ in the $-1.50 \mathrm{D}$.In the case of -1.00 and $-4.75 \mathrm{D}$ lenses there is an increase of $26 \%$.

Conclusions: The ionic permeability of silicone hydrogel lenses of different optical powers was not significantly different. Worn lenses present a significant reduction of the ionic permeability after a month of wear.

KEYWORDS: Silicone Hydrogel; hydrogel; contact lenses; ionic permeability; ionoflux. 


\section{INTRODUCTION}

The ionic permeability of contact lenses to salt, is regarded by some manufacturers as a quality control parameter of the material. This parameter refers basically to the ability of the $\mathrm{Na}^{+}$and $\mathrm{Cl}^{-}$ions have to pass through the polymeric matrix of the contact lens and it is believed that the movement of the contact lens over the ocular surface is affected when this assumes values lower than $2.4 \times 10^{-7} \mathrm{~cm}^{2} / \mathrm{s}{ }^{1}$ However, there is little information in the state of the art available concerning this subject and particularly the effect of lens wear on this property.

Yasuda et al. measured aqueous $\mathrm{NaCl}$ permeability $(P)$, diffusion coefficients $(D)$, and equilibrium partition coefficients $(k)$ for crosslinked hydrogels. They verified that the salt diffusivity and partition coefficient increased as the membrane water content $(w)$ increased. In agreement with free-volume theory, the $\mathrm{NaCl}$ increased monotonically with membrane water content once the partition coefficient varied linearly with water volume fraction for high-water-content materials. Yoon and Jhon ${ }^{2}$ investigated Hydroxyethyl methacrylate (HEMA)-based membranes with different crosslink densities and confirmed the results obtained by Yasuda et al. ${ }^{3}$

Hamilton et al measured the permeability of aqueous strong electrolytes (including $\mathrm{NaCl}$ ) through poly(hydroxyethyl methacrylate) (PHEMA) and pHEMA/methyl methacrylate copolymer membranes. Their results demonstrated that salt permeability increase with the increase of hydrogel water content. They established that the influence of ion size, ion and polymer water structure also contribute to salt permeability in hydrogels. ${ }^{4}$ Nicolson et al. argue that water is compressed out of a contact lens during a blink and replenished during an interblink period. ${ }^{5}$ The squeezedout water maintains a thick enough post-lens tear film which avoids the contact lens adhesion on ocular surface. Therefore, a threshold water hydraulic permeability is 
necessary to maintain lens movement. These authors also suggested that the salt permeability indirectly reflects water transport rate through a soft contact lens, which is also a relevant parameter when it comes to understand the dynamics of solutes and gas molecules across the lens material. Tighe, with a simple calculation demonstrated that to maintain an adequate PoLTF boundary layer thickness is necessary a critical hydraulic permeability. ${ }^{1}$ Monticelli et al. ${ }^{6}$ disagree with the need of a squeeze-out nor a squeeze-through mechanism to maintain the PolTF because hydraulic permeability of soft contact lens are tiny. ${ }^{6}$

Domschke et al. $^{7}$ and later Nicolson and $\operatorname{Vogt}^{5}$ argue an alternate explanation to critical salt permeability of soft contact lens. These authors suggested that continuous water paths disconnect below critical water content in a hydrogel. A percolation model distinguishes lens material that bind on the cornea to those that will move over the ocular surface as required. ${ }^{1 ; 8}$ Recent measurements of salt permeability of low water content silicone hydrogel material demonstrated a continuous decline with decreasing water content down to $10^{-9} \mathrm{~cm}^{2} / \mathrm{s}$ at $0.2 \mathrm{wt} \%$ water which was well below the cited threshold necessary for on-eye movement. ${ }^{9}$

To our knowledge, no previous study has evaluated the potential effect of lens wear on the ionic permeability of contact lens polymers. With the present work we aimed to test the hypothesis that soiling and degradation of the material polymers could affect the ionic permeability of current contact lens materials. 


\section{MATERIAL AND METHODS}

Currently, there are two methods to measure the ion permeability, Ionoflux and Ionoton methods. The ionoflux method has been used in the present work and will be described in more detail in this section. The ionoton method will be presented briefly in the discussion section.

In general the Ionoflux technique is a preferred method for determining the ion permeability of a lens that allows to determine the probability of adequate on-eye movement according to the Patent EP0819258B1. ${ }^{10}$ Ionoflux Technique use a conductimeter, an electrode with a temperature sensor, a donor chamber containing a salt solution, a receiving chamber containing milli-Q water (ultrapure water), a stir bar and a thermostat. The donor chamber is specially designed for sealing and securing contact lens. This has a glass tube which is threaded at the end and the glass tube.

The donor chamber has a sealing function of the contact lens and so that the donor solution does not pass around the lens (i.e., ions may only pass through the lens). It is constituted by a glass tube threaded at the end which is immersed in the receiving solution. The glass tube presents a centrally located aperture of about $9 \mathrm{~mm}$ in diameter. A lid, which is threaded to mate with the glass tube, holds a lens-retaining member which includes a centrally located hole of about $8 \mathrm{~mm}$ in diameter. The lens-retaining piece contains a male portion adapted to mate with and seal the edges of inner (concave) surface of lens and female portion adapted to mate with seal the edges of the outer (convex) surface of a lens.

In the lens-retaining piece the lens to be measured is placed between the male and the female portions which contain flexible sealing rings. When the lens is in the lens-retaining piece, the same device is placed in the threaded lid and this screwed onto 
the glass tube to define the donor chamber. Then, the donor chamber is filled with 16 $\mathrm{mL}$ of $0.1 \mathrm{M} \mathrm{NaCl}$ solution. The receiving chamber is filled with $100 \mathrm{~mL}$ of milli-Q water (ultrapure water). The leads of the conductivity meter are immersed in the milli-Q water of the receiving chamber and a stir bar is added to the receiving chamber. This chamber is positioned in a thermostat and the temperature is held at about $35^{\circ} \mathrm{C}$. Finally, the donor chamber is immersed in the receiving chamber.

During each measurement trial, the conductivity measurements are taken every minute for 40 minutes, starting 10 minutes after immersion of the donor chamber into the receiving chamber. (TANTO TIEMPO)

\section{Calibration of the Conductivity Electrodes}

The electrode's conductivity is calibrated beforehand. The calibration curve was obtained at different concentrations of salt and milli-Q water $(18.2 \mu \mathrm{S} / \mathrm{cm})$. The solutions used in the calibration had the following concentrations of $\mathrm{NaCl}, 10^{-1}, 10^{-2}, 10^{-}$ ${ }^{3}, 5 \times 10^{-4}, 10^{-4}$ and $10^{-5} \mathrm{M}$. The conductivity of each solution was measured with the electrode and its values are plotted for each concentration.

\section{Ion Permeability Measurements}

The ion permeability of a silicone hydrogel contact lens can be determined from the rate of ion penetration through the lens, from one surface of the lens to another according to the experimental device shown in the Figure 1. The technical details of the contact lens used in this study are presented in table 1. 
Table 1. Technical details of the contact lens used in this study

\begin{tabular}{|l|l|}
\hline Trade name & Biofinity \\
\hline Manufacturer & Coopervision \\
\hline Material (USAN) & Comfilcon A \\
\hline EWC (\%) & 48 \\
\hline Dk (barrer) & 128 \\
\hline Dk/t (-3.00D) & 160 \\
\hline Power (D) & -3 \\
\hline Base Curve (mm) & 8.6 \\
\hline Diameter (mm) & 14.0 \\
\hline
\end{tabular}

In this device of the lens is positioned between two solutions contained in chambers with known and different ionic concentrations salts. In compartment A (the donor chamber), there is a solution of $\mathrm{NaCl} 1 \mathrm{M}$ and in the compartment $\mathrm{B}$ (receiver chamber), milli-Q water. In compartment $\mathrm{B}$, is positioned an ion selective $\mathrm{Na}$ electrode (Model EC meter GLP31) for measuring the conductivity in a time function due to the amount of ions reaching the reservoir after passing through the contact lens.

The sensor is attached with a temperature sensor to control the temperature over time inside compartment B. The compartment B (receiver chamber) has a volume of $100 \mathrm{~cm}^{3}$. A stirring magnetic bar to make constant agitation (800 rpm) and a thermostat to ensure that the temperature is constant during the measurements were also placed inside Compartment B. In one of the experiments, a smaller agitator is placed on the donor chamber to evaluate the influence of this process on the final result. The chamber A (donor chamber) has a volume of $16 \mathrm{~cm}^{3}$ and it is designed to perfectly seal a lens so that the donor solution doesn't go around the lens (and ions can only pass through 
exposed contact lens). The camera consists in a teflon tube which contain a hole of 7 mm diameter (Figure 1).

The retainer includes a male portion adapted (concave) to coincide with the lens, and a female portion adapted to mate and seal the edges of the outer surface (convex) lens. Finally the lens to be measured is placed between the male and female portions of the cell and the Teflon tube is screwed so that the lens is completely sealed in the donor chamber. Once the lens is placed, it is filled with a volume of $16 \mathrm{~cm}^{3}$ of saline solution and immersed in $100 \mathrm{~cm}^{3}$ of milli-Q water of the receiving chamber. Then it is added a magnetic stir bar to homogenize the solution under the lens and further decrease the resistance of water layers. The conductivity meter and magnetic stir bar was placed in the receiving chamber that is thermostatically controlled by thermostat so that the experience is carried out at $35^{\circ} \mathrm{C}$.

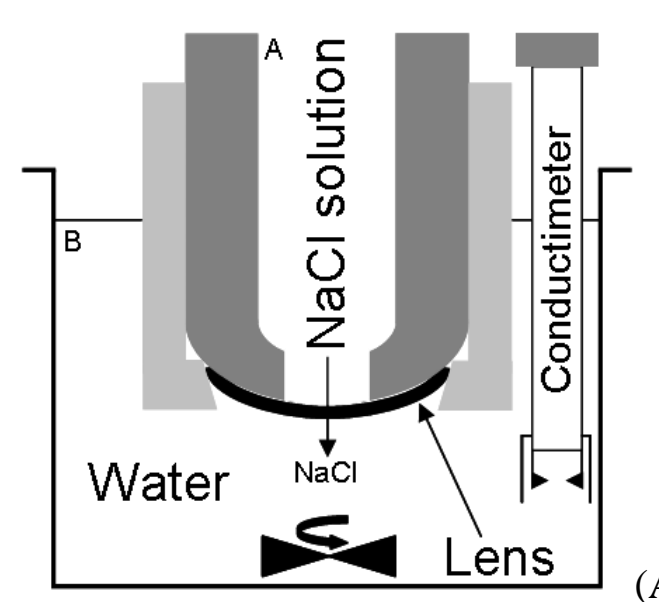

(A)

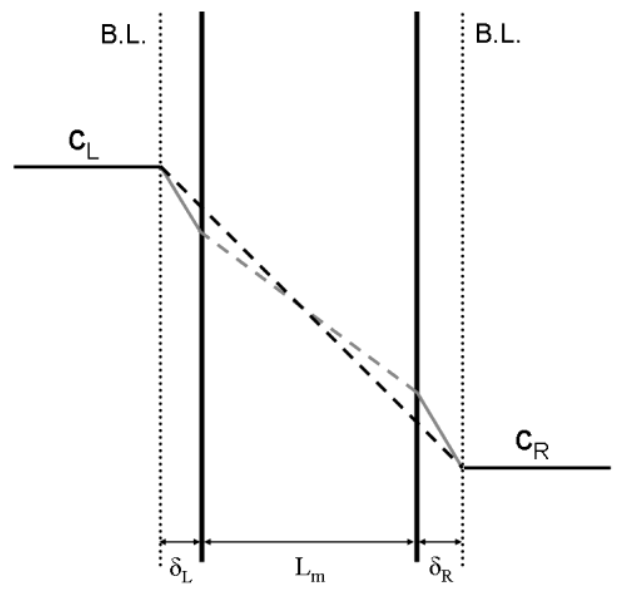

Figure 1. (A) Schematic representation of the experimental set-up used for the determination of the methanol permeability across the membranes. The potentiometric method is specifically represented. (B) Scheme of the permeation phenomenon across a membrane. The gray profile represents the real process occurring, while the black discontinuous line represents the apparent permeability of the membrane by neglecting the boundary layer (B.L.) effects. The gray discontinuous line inside the membrane shows the true (intrinsic) permeability profile. 
Worn contact lenses made of one of the same material (Comfilcon A) were measured and then compared to new samples from the same batch in order to evaluate the effect of lens wear on ionic permeability. Additionally, these lenses had different optical powers $(-1.00,1.50$ and -4.75$)$ allowing us to evaluate the effect of refractive power and its thickness profiles in ionic permeability measures.

\section{Extraction of Ionic Permeability and Related Parameters}

The flux of salt $\mathrm{J}$ can also be expressed as the amount of $\mathrm{NaCl}$ in moles $n$ crossing the membrane (of harmonic average thickness L) per unit of time $t$ and area $A$, is given by the first Fick's law as

$$
J=\frac{d n}{A d t}=D_{m} \frac{\partial C_{m}}{\partial x}=D_{m} k_{m} \frac{\Delta C}{L}=P \frac{\Delta C}{L}
$$

where $\Delta C$ represents the variation of salt concentration between the right and left side of the membrane, with a average thickness $\mathrm{L}$, and can be expressed as $C_{B^{-}} C_{A} . D_{m}$ is the salt diffusion coefficient into the membrane, $P$ is the apparent permeability coefficient of the sodium ion $\left(\mathrm{P}=\mathrm{D}_{\mathrm{m}} \mathrm{k}_{\mathrm{m}}\right)$, and $\mathrm{k}_{\mathrm{m}}$ is the lens salt partition coefficient.

Integrating equation 1 , the following expression is obtained ${ }^{3}$ :

$$
\ln \left(1-\frac{2 c_{B}(t)}{c_{A, 0}}\right)=-\frac{2 \cdot A \cdot P}{V_{B} \cdot L}\left(t-t_{0}\right)
$$

Where $c_{B}(t)$ is the concentration of sodium ions at time $t$ in the receiving chamber, $c_{A, 0}$ is the initial concentration of sodium ions in donor chamber, $A$ is the area of the lens exposed to the salt flux, $L$ represent the average lens thickness in the area exposed and $V_{B}$ is the volume of the receiving cell compartment (magnitudes that are known),

From eq. (2), the salt concentration in the receiver compartment in case of $c_{B}<<c_{A, 0}$, as it happens in our experiments, will be, 
$c_{B}=\frac{A \cdot P \cdot c_{A, 0}}{V_{B} \cdot L}\left(t-t_{0}\right)$

Being $\mathrm{c}_{\mathrm{A}, 0}$ the initial concentration of salt in the chamber where the distilled water has been introduced and the salt conductivity is measuring, and $t_{0}$ represent the time lag.

Thus, plotting the concentration of $\mathrm{Na}^{+}$ions in chamber $\mathrm{B}$ versus time allows us to obtain the apparent $\mathrm{Na}^{+}$permeability of the lens from the slope of eq.(3). When the lens has high solute sorption capacity, there will be a time lag before the pseudo-steady state is reached in the permeation experiment. From the curve of concentration vs. time, the time lag $t_{0}$ can be obtained from the intersection of pseudo-state line with the $\mathrm{x}$-axis representing time. ${ }^{9}$ The diffusion coefficient can be then estimated as:

$D=\frac{L^{2}}{6 t_{0}}$

Finally the tortuosity, $\tau$, of each hydrogel can be estimated from the relation ${ }^{11}$

$$
D_{0}=D \cdot \tau^{2}
$$

Where $D_{0}$ is the molecular self-diffusion coefficient of aqueous sodium chloride $\left(2.089 \times 10^{-5} \mathrm{~cm}^{2} / \mathrm{s}\right.$ at $\left.35^{\circ} \mathrm{C}\right) .{ }^{12}$

\section{RESULTS}

The conductivity measured for each $\mathrm{NaCl}$ solutions prepared for calibration curve has been plotted vs. concentration of salt. The calibration curve obtained for almost four orders of magnitude of salt concentrations is shown in figure 2. The inset represent a zoom which correspond to low concentrations (less than $0.01 \mathrm{M}$ concentration of $\mathrm{NaCl}$ ). The conductivity shift has a linear correlation with $\mathrm{NaCl}$ concentration with a slope of $1222 \pm 39 \mathrm{mS}$ (miliSiemens). 


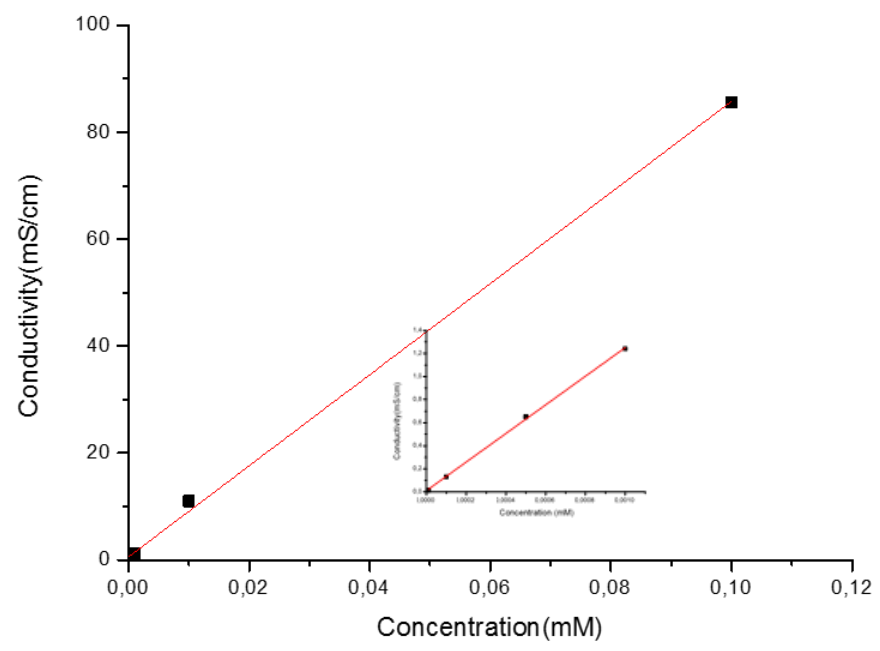

Figure 2. Calibration curve for the conductivity of $\mathrm{NaCl}$ (aq) solutions versus $\mathrm{NaCl}$ concentration. The inset graphic represents conductivity versus $1 \times 10^{-5}, 1 \times 10^{-4}, 5 \times 10^{-4}$, $1 \times 10^{-3} \mathrm{NaCl}$ concentrations. The large graphic represents conductivity versus $1 \times 10^{-5}, 1$ $\times 10^{-4}, 5 \times 10^{-4}, 1 \times 10^{-3}, 1 \times 10^{-2}$ e $1 \times 10^{-1} \mathrm{NaCl}$ concentrations.

Measurement of new and worn lenses of different optical powers showed significant differences in ionic permeability as results of lens wear. Table 2 shows the values of permeability and harmonic lens thickness (measured over 5 different points across the lens central $8 \mathrm{~mm}$ ).

Table 2. Ionic Permeability (P) and harmonic lens thickness (L) of new and worn Biofinty contact lens $(-1.00,-1.50,-4.75 \mathrm{D})$

\begin{tabular}{|l|ll|l|}
\hline Power & $\mathbf{P}\left(\mathbf{1 0}^{\mathbf{8}} \mathbf{c m}^{\mathbf{2}} / \mathbf{s}\right)$ & $\mathbf{L} \mathbf{( 1 0} \mathbf{m m})$ \\
\hline \multicolumn{3}{|c|}{ New Biofinity } \\
\hline-1.00 & $10.0 \pm 1.6$ & $1.19 \pm 0.01$ \\
\hline-1.50 & $11.50 \pm 0.12$ & $1.12 \pm 0.02$ \\
\hline-4.75 & $12.6 \pm 1.0 \quad$ Used Biofinity \\
\hline \multicolumn{3}{|c|}{$0.99 \pm 0.01$} \\
\hline-1.00 & $7.4 \pm 0.2 \quad 1.14 \pm 0.05$ \\
\hline-1.50 & $7.1 \pm 0.2$ & $1.04 \pm 0.02$ \\
\hline-4.75 & $8.8 \pm 0.5$ & $1.04 \pm 0.08$ \\
\hline
\end{tabular}

The value of apparent permeability to all powers, both new and used is represented in figure 3. A brief observation of these values allows us to infer that the 
new lenses shows greater coefficient permeability than the used lenses. $P_{\text {ion }}=10^{-9} \mathrm{~cm}^{2} / \mathrm{s}$ and this critical value required to ensure that the lens can move on the eye.

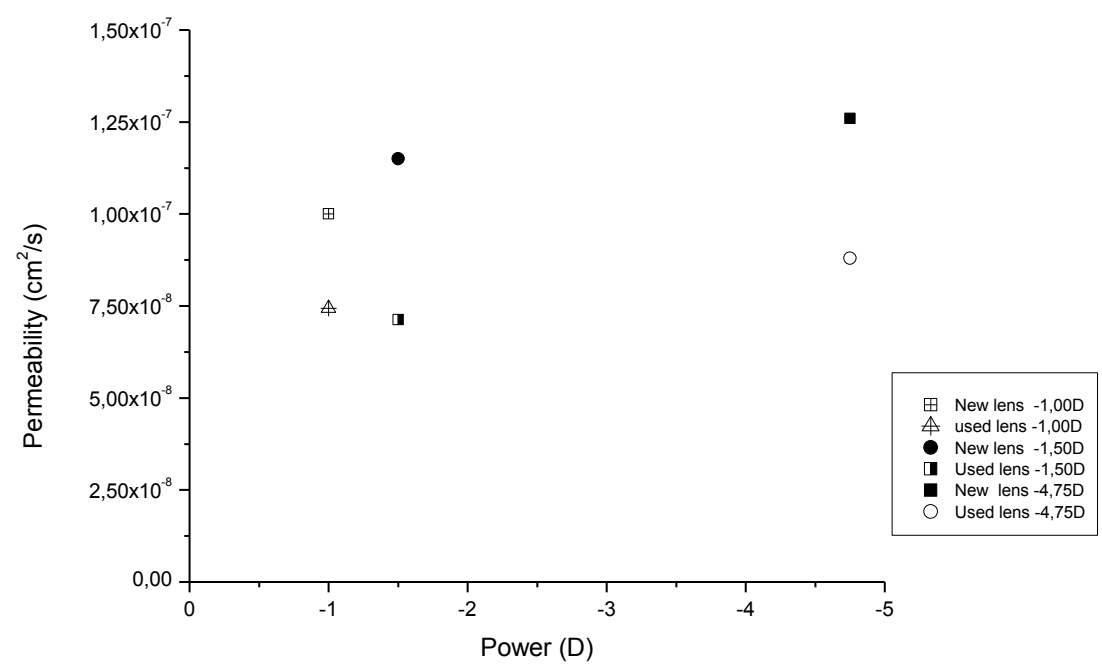

Figure 3. Ionic permeability for new and used lenses of three different optical powers ($1.00,-1.50$ and $-4.75 \mathrm{D}$.

\section{DISCUSSION}

According with Domschke et al. the ion permeability of materials used as contact lenses is a critical parameter for the lenses because it permit predict the motion of the lens when it is situated onto the corneal surface. ${ }^{7}$ The effect of lens optical power and used lens in the ionic permeability has never been studied before.

Taking in account the values obtained for the ionic permeability and considering that this coefficient is a characteristic parameter intrinsic of each lens, we can conclude that the permeability of the new Biofinity lens to sodium ions is equal to $(11.4 \pm 1.1)$ $\times 10^{-8} \mathrm{~cm}^{2} / \mathrm{s}$ while the used lens is equal to $(7.8 \pm 0.7) \times 10^{-8} \mathrm{~cm}^{2} / \mathrm{s}$ which means that the 
ionic permeability of the used lenses decreases $31 \%$ when it is compared to new lenses will.

We can connect the decrease of ionic permeability in used contact lens with dehydration lens and deposits on their surfaces. The evaporation present during contact lens wear and electro-osmotic drag processes have the potential to severely diminish the contact lens hydration. If the hydration of the contact lens decreases the ionic permeability also will be decreased because the $\mathrm{NaCl}$ transport occurs in the water phase of the gel.

If one compares the apparent ionic permeability between lenses with -1.00 and $1.50 \mathrm{D}$, we can observe that there is an increment in the $-1.50 \mathrm{D}$ lenses of $15 \%$.

In the case of -1.00 and $-4.75 \mathrm{D}$ lenses there is an increase of $26 \%$. These values were not the expected because this parameter should be an intrinsic parameter characteristic of the material. Wolffsohn et al., demonstrated that together with centration and limbal incursions, horizontal lag, movement on blink in up-gaze and push-up recovery speed should be recorded to adequately describe soft contact lens fit. ${ }^{13}$

This study demonstrated that there is more to understand about ionic permeability of soft contact lenses. In future studies, we suggest that would be important the simultaneous evaluation of ionic conductivity and on-eye lens performance; measure ionic permeability in high myopic lens (above -6.00D) and positive lens (thicker at the lens center might be worse this parameter); evaluate if the ionic permeability decrease is accompanied by changes in the dynamic behavior of the contact lenses on the eye.

There is another method to measure the ionic permeability of hydrogel membranes. In recent work, Guan et al. ${ }^{8}$ measured the aqueous permeability, partition 
coefficient, and diffusion coefficient for commercial soft contact lens under physiological conditions using the. They used the Ionoton method in a miniature Stokes cell and demonstrated that the results obtained was similar to the values obtained with the Ionoflux method. The permeability increased exponentially with water content above a certain level. The partition is the ratio of concentrations of a compound in a mixture of two immiscible phases at equilibrium. This increased approximately linearly and the diffusivities decreased exponentially with increase in water content according to free-volume theory. They did not observe differences between the $P, k_{m}$, or $D_{m}$ behavior for aqueous $\mathrm{NaCl}$ diffusion between HEMA-based and siloxane-based soft contact lenses. $^{8}$

In summary, from this study we can conclude that stirring on the donor chamber does not have a significant effect on the measurement obtained. Differences between stirring and non-stirring were in the order of less than 5\%. Similar results were obtained when the lens first were equilibrated with a solution of $\mathrm{NaCl} 1 \mathrm{M}$ or with ultrapure water. However, some lenses could not be measured in either condition. The ionic permeability of new and used myopic contact lens of different optical powers -1.00D, 1.50D and $-4.75 \mathrm{D}$ were also measured. From these measurements we can observe that Lens already used affects the ionic permeability in about 25 to $38 \%$. Finally, lenses with different thicknesses have different ionic permeability, with a significant reduction for moderately myopic lenses around -5.00 as measured in this study. 


\section{REFERENCES}

\section{Reference List}

1. Tighe B. Silicone hydrogel materials-How do they work? In: Sweeney DF, ed. Silicone hydrogels. The rebirth of continuous wear contact lenses. Oxford: Butterworth-Heinemann; 2000:1-21.

2. Yoon SC, Jhon MS. The transport phenomena of some model solutes through postcrosslinked poly(2-hydroxyethyl methacrylate) membranes with different tactic precursors. J Appl Polym Sci 1982;27:3133-49.

3. Yasuda H, Lamaze CE, Ikenberry LD. Permeability of solutes through hydrated polymer membranes. Part I. Diffusion of sodium chloride. Makromol Chem $1968 ; 118: 19-35$.

4. Murphy SM, Hamilton CJ, Tighe BJ. Synthetic hydrogels: 5. Transport processes in 2-hydroxyethyl methacrylate copolymers. Polymer 1988;29:188793.

5. Nicolson PC, Vogt J. Soft contact lens polymers: an evolution. Biomaterials $2001 ; 22: 3273-83$

6. Monticelli MV, Chauhan A, Radke CJ. The effect of water hydraulic permeability on the settling of a soft contact lens on the eye. Curr Eye Res 2005;30:329-36. 
7. Domschke A, Lohmann D, Winterton L. On-eye mobility of soft oxygen permeable contact lenses. Proceedings of the ACS Spring National Meeting SanFrancisco: PMSE. 1997.

Ref Type: Abstract

8. Guan L, Jim+®nez MEG, Walowski C, et al. Permeability and partition coefficient of aqueous sodium chloride in soft contact lenses. J Appl Polym Sci 2011;122:1457-71.

9. Cheng ML, Sun YM. Observation of the solute transport in the permeation through hydrogel membranes by using FTIR-microscopy. Journal of Membrane Science 2005;253:191-8.

10. Baron RC, Chabrecek P, Court J, et al. Extended wear ophthalmic lens. 9-122001. Google Patents.

Ref Type: Generic

11. Chhabra M, Prausnitz JM, Radke CJ. A single-lens polarographic measurement of oxygen permeability (Dk) for hypertransmissible soft contact lenses. Biomaterials 2007;28:4331-42.

12. Aseev GG. Electrolyte, Transport Phenomena: Methods for Calculation of Multicomponet Solutions and Experimental Data on Viscosities and Diffusion Coefficients. New York: Begell House; 1998.

13. Wolffsohn JS, Hunt OA, Basra AK. Simplified recording of soft contact lens fit. Cont Lens Anterior Eye 2009;32:37-42. 
Ionic Permeability of Worn Contact Lenses 